\title{
Synaptogenesis in the Rat Suprachiasmatic Nucleus Demonstrated by Electron Microscopy and Synapsin I Immunoreactivity
}

\author{
Robert Y. Moore ${ }^{1,2}$ and Mary E. Bernstein 1 \\ Departments of ${ }^{1}$ Neurology and ${ }^{2}$ Neurobiology and Behavior, State University of New York at Stony Brook, Stony Brook, \\ New York 11794
}

\begin{abstract}
Synaptogenesis was studied in the rat suprachiasmatic nucleus (SCN) using quantitative ultrastructural analysis and synapsin I immunohistochemistry. SCN neurons are formed between embryonic days 13 and 17 (E13-E17), and the SCN is a distinct hypothalamic nucleus by E18. At E19 the nucleus is very immature and there are few synapses. Synaptogenesis proceeds slowly until P4 but increases rapidly between $P 4$ and P10. At P10 the number of synapses per unit area is the same as in the adult $\mathrm{SCN}$ and all synaptic types present in the adult are evident. However, the SCN continues to increase in volume to the adult with approximately $30 \%$ of total synaptic number added between P10 and a young adult age. The appearance of synapsin I immunoreactivity correlates very precisely with the development of synapses in the SCN as shown by ultrastructural analysis between E19 and P6. The pattern of appearance of synapsin I immunoreactivity demonstrates that synaptogenesis in the SCN is significantly delayed in comparison to adjacent hypothalamic nuclei. Synapsin I immunohistochemistry is a reliable marker of synapse formation in the developing SCN. A correlation of these anatomical data with prior functional studies suggests that SCN neurons are born as individual circadian oscillators that undergo a rapid development in the first $\mathbf{1 0}$ days after birth to form a functional neural network subserving circadian rhythm generation and regulation.
\end{abstract}

The suprachiasmatic nucleus (SCN) is a circadian pacemaker in the mammalian brain (Rusak and Zucker, 1979; Moore, 1983; Turek, 1985; Rosenwasser and Adler, 1986). Although circadian rhythms typically develop postnatally, the rhythm in glucose metabolism demonstrated by the 2-deoxyglucose (2-DG) method (Schwartz and Gainer, 1977; Schwartz et al., 1980; Sokoloff, 1982) develops prenatally (Reppert and Schwartz, 1983, 1984), shortly after the formation of the $\mathrm{SCN}$ from the ventral diencephalic germinal epithelium (Ifft, 1972; Altman and Bayer, 1978,1986 ). It has generally been considered that much of the functional activity demonstrated by the 2-DG method reflects synaptic activity (Sokoloff, 1982), but prior studies of SCN development indicate that the nucleus is immature prenatally and

\footnotetext{
Received Aug. 17, 1988; revised Nov. 2, 1988; accepted Nov. 18, 1988.

This work was supported by NIH Grant NS-16304. We are grateful to Faye Eldred, Ioan Speh, and Priscilla Wu for skilled technical assistance. The manuscript was prepared while one of the authors (R.Y.M.) was a Fellow-in-Residence, The Neurosciences Institute, Neurosciences Research Program, New York, NY.

Correspondence should be addressed to Dr. Robert Y. Moore, Department of Nemology, HSC, T-12, SUNY at Stony Brook, Stony Brook, NY 11794.

Copyright (C) 1989 Society for Neuroscience $0270-6474 / 89 / 062151-12 \$ 02.00 / 0$
}

that synaptogenesis is predominantly a postnatal event (Lenn et al., 1977; Koritsanszky, 1981). However, neither of those studies provided more than a brief, qualitative analysis of SCN development. For that reason the present study was undertaken to obtain a detailed, quantitative ultrastructural analysis of synaptogenesis in the $\mathrm{SCN}$ and correlate this with the development of immunoreactivity to a specific synaptic protein, synapsin I (DeCamilli et al., 1979, 1983a, b).

\section{Materials and Methods}

Animals and surgery. Pregnant female albino rats of the Sprague-Dawley strain were obtained from the dealer (Taconic) at $7-10 \mathrm{~d}$ gestation (timed pregnancy). They were maintained in individual cages under standard laboratory conditions in diurnal lighting (lights on 0700-1900 hr) with free access to food and water. In our laboratory these rats typically give birth on the 22nd day after mating. The day after mating is designated embryonic day 1 (E1). For studies of fetuses, pregnant females were anesthetized with pentobarbital $(80 \mathrm{mg} / \mathrm{kg})$, and fetuses were removed from the uterus and perfused. Any fetus that showed a response to stimulation received additional pentobarbital. The age of the fetus was confirmed by measurement of crown-rump length as described by Seiger and Olson (1973). The day of birth was designated P1. All postnatal animals were anesthetized with pentobarbital $(80 \mathrm{mg} / \mathrm{kg})$ prior to being killed. The numbers of animals used are noted below.

Electron microscopy. For the electron microscopic analysis, 26 animals of both sexes were used at ages E19 (4 animals), E21 (4), P1 (3), P2 (3), P4 (4), P6 (3), P10 (3), and adult (2). Animals at E19-P4 were perfused with physiological saline followed by $1 \%$ paraformaldehyde$1.5 \%$ glutaraldehyde in $0.1 \mathrm{~m}$ Sorenson's buffer ( $\mathrm{pH} 7.4$ ). The brains were immersed in $2 \%$ paraformaldehyde- $2.5 \%$ glutaraldehyde with agitation for $\mathbf{4 h r}$ at room temperature, blocked, and refrigerated overnight. Following a buffer rinse, the tissue was osmicated, dehydrated, and embedded in Spurr resin. P6, P10, and adult animals were perfused with saline followed by $2 \%$ paraformaldehyde- $2.5 \%$ glutaraldehyde in $0.1 \mathrm{~m}$ Sorenson's buffer, blocked, and fixed overnight in the same fixative. Blocks were rinsed in buffer, osmicated, dehydrated, and embedded in Epon-Araldite.

Ultrathin coronal sections from middle rostrocaudal levels of the SCN were mounted on Formvar-coated slot grids, stained with uranyl acetate and lead citrate, and examined with a JEOL $100 \mathrm{CX}$ electron microscope. Capillary maps from toluidine blue stained, $0.5 \mu \mathrm{m}$ sections adjacent to each group of thin sections permitted precise localization of each micrograph. Micrographs for axon terminal counts were chosen nonsystematically from several rostrocaudal levels of the nucleus in each brain, photographed at $3300 \times$, enlarged 5 times, and analyzed using a $2 \times$ or $3 \times$ loupe to give a final magnification of 33,000 or 49,000 . Low magnification was used to reduce bias in area selection, and the micrographs included perikarya, blood vessels, and axon bundles. Thus, the counts represent numbers of axon terminals in SCN tissue rather than in neuropil. At older ages, a portion of the $\mathrm{SCN}$ is interdigitated with the dorsal optic chiasm, but this area was not analyzed to maintain methodological consistency with younger ages. Axon terminals are defined as enlarged components of the axon containing accumulations of synaptic vesicles. Very few axon terminals are evident at E19-P2, and those present are often immature. Hence, for E19-P2 an axon terminal 


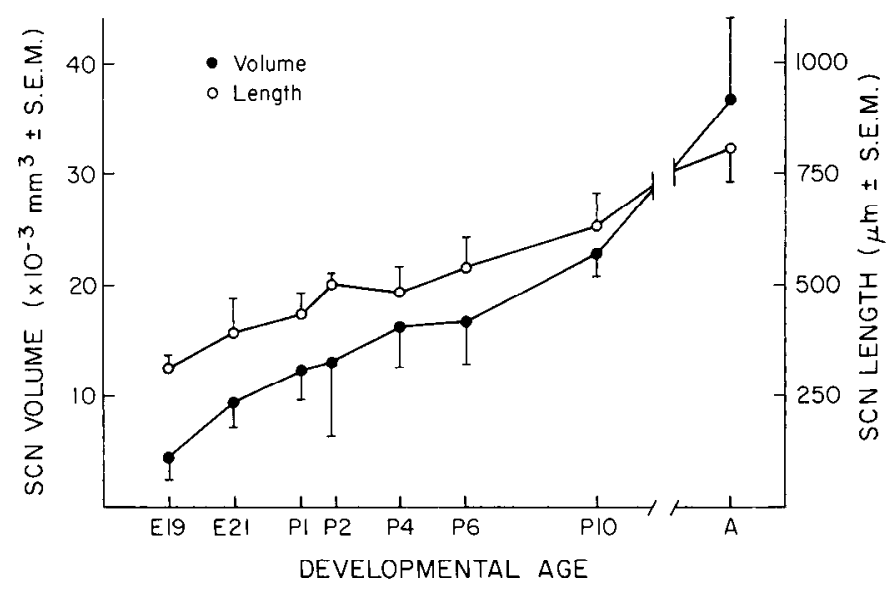

Figure 1. Development of SCN length and volume. The length and volume of the nucleus are shown as a function of developmental age.

was counted if there was an evident membrane thickening and 3 or more synaptic vesicles nearby. $\Lambda \mathrm{t} \mathbf{P} 4$-adult the boutons are mature with typical accumulations of vesicles. In addition to a total synaptic count, the nucleus was subdivided into 4 quadrants-dorsomedial, ventromedial, dorsolateral, and ventrolateral-and counts were made from each quadrant at each developmental age. This division was made because visual afferents to the SCN terminate predominantly in the ventral and lateral quadrants.

Synapsin I immunohistochemistry. Twenty-eight animals of both sexes at ages E19(4), E21(3), P1(4), P2(3), P4(3), P6(3), P8(3), P10(2), and adult (1) were perfused transcardially with $0.1 \mathrm{M}$ PBS followed by Bouin's fixative. Brains were processed for paraffin embedding and $10 \mu \mathrm{m}$ coronal sections through the hypothalamus were cut. Every 10th section was mounted for histochemistry and the adjacent section was mounted for staining with cresyl violet. The histochemical material was prepared using the peroxidasc-antipcroxidase method. Synapsin I antiscrum was generously provided by Drs. Paul Greengard and Charles Ouimet (The Rockefeller University, New York, NY). It is a well-characterized antiserum (DeCamilli et al., 1983a, b), and preliminary data demonstrated that it provides equally good reactions on frozen and paraffin-embedded sections. Details of the immunohistochemical methodology have been provided previously (Card et al., 1981). The cresyl violet-stained series was used to determine the boundaries of the SCN in the immunohistochemical material and for morphometric analysis. In addition to the material noted above, 2 brains each from E16 and E17 were sectioned in the coronal plane and stained with cresyl violet to study the early development of the SCN. Morphometric analyses of SCN length and volume were made using a Bioquant image analysis system.

\section{Results}

SCN development

General features

The major focus of this study was synaptic development in the $\mathrm{SCN}$, but it is necessary to put this in a context of the general development of the nucleus. Nissl-stained material was analyzed from E16 to P10. At E16 the germinal epithelium in the anterior hypothalamic area is quite thick with numerous mitotic figures. No clearly defined SCN is apparent. At E18 activity in the germinal epithelium is minimal and the SCN is clearly defined, containing closely packed small neurons. There are 2 major changes evident in Nissl-stained material that evolve from E19 to P10. First, the nucleus becomes progressively larger, as does the rest of the brain. Second, SCN neurons become increasingly less densely packed with the apparent amount of neuropil increasing. Even so, it is worth noting that the SCN contains the highest perikaryal density of any hypothalamic nucleus, even in the adult brain. The increase in size of the nucleus is readily apparent from measurements of length and volume (Fig. 1). These gradually increase at a nearly identical rate from E19 through P10. It should be noted that a small increase in length and a marked increase in volume occurs after P10. This presumably represents an increase in total neuropil as the number of synapses per unit area does not increase (see below).

In material prepared for electron microscopy, the general development of the SCN conforms to what would be inferred from the Nissl material. At E19 the SCN contains numerous small, immature neuronal somata that are closely approximated and often in strands or rings. The neuropil is sparse and contains predominantly large and medium-sized dendritic profiles and dendritic growth cones (Fig. $2 A$ ). There are occasional bundles of small, unmyelinated axons and a few glia. A few radial glial processes with opposed neuronal perikarya are also present. Perikarya throughout the nucleus are small with sparse cytoplasm and only occasional cytoplasmic invaginations into the nucleus. We do not observe gap junctions between neurons at E19 or any other age in our material, but we did not make a systematic search for them. Tight junctions, typical puncta adherens, are present in fetal material (Fig. $2 B$ ) and occasionally found in the postnatal SCN. Between E19 and P10 the major change in SCN ultrastructure is in the development of the neuropil. Neuronal perikarya become more dispersed, although occasional strands or rings of closely opposed somata are still present in the adult SCN. At E21 neuronal cytoplasm is noticeably more dense than at E19 because of a marked increase in the number of ribosomes. Neuronal cytoplasm continues to mature until P10, when the neurons have an essentially adult appearance. In the adult, there are 2 types of neurons that have a preferential distribution in the SCN. The first comprises small neurons with scant cytoplasm and few, simple nuclear invaginations. These are found predominantly in the dorsomedial part of the nucleus. The second comprises larger neurons with more extensive cytoplasm and complex nuclear invaginations, which are located primarily in the ventrolateral portion of the nucleus. The distinction is not evident in early development and becomes clear only between P6 and P10. Neurons at E19-P2 exhibit perikaryal budding so that formation of primary dendrites is completed by $\mathrm{P} 4$. Cell death occurs in the $\mathrm{SCN}$ from E21 through P6. The number of degenerating perikarya is small at any stage, and degenerating perikarya are nearly always observed as single neurons, rarely as members of clusters or strands. Since the time course over which the changes occur is unknown, the importance of cell death in the development of the SCN cannot easily be assessed.

The neuropil becomes progressively more complex between E21 and P10 (Figs. 3 and 4). The number of dendritic and axonal profiles increases as does the number of synapses (see below). More small dendrites and dendritic spines are evident. In addition, there are increasing numbers of small, unmyelinated axons, particularly in small fascicles, present in all regions of the SCN. The development of the neuropil is such that it appears mature in all parts of the nucleus except at the interface between the optic chiasm and the SCN by P6. A general feature of SCN synaptology is that a large proportion of synapses are on small dendritic branches and dendritic spines. Axosomatic synapses are present but uncommon. Dendrodendritic synapses are present, particularly in the ventral portion of the nucleus, but these also are not common. A prominent feature of the ventral neuropil in the adult is an arrangement of small dendrites and axons, particularly those of retinal origin, in a glomerular organization. 

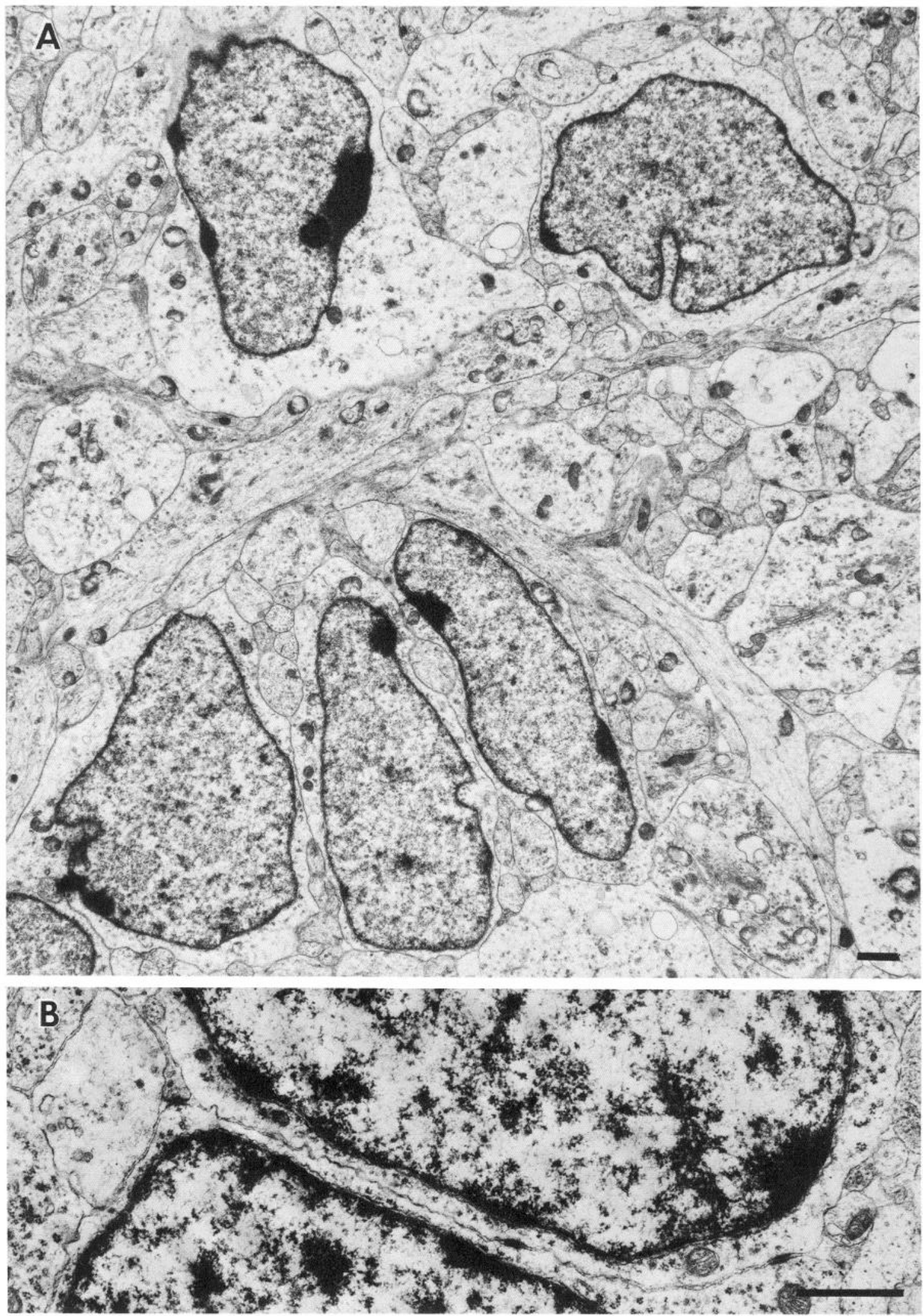

Figure 2. Electron micrographs showing SCN ultrastructure at E19. A, Neurons and neuropil. The neuronal perikarya are small with a scant cytoplasm containing few organelles. The neuropil contains dendrites, small axons, and some growth cones. Scale bar, $5 \mu \mathrm{m}$. $B$, Two adjacent neurons with tight junctions. Scale bar, $2 \mu \mathrm{m}$. 


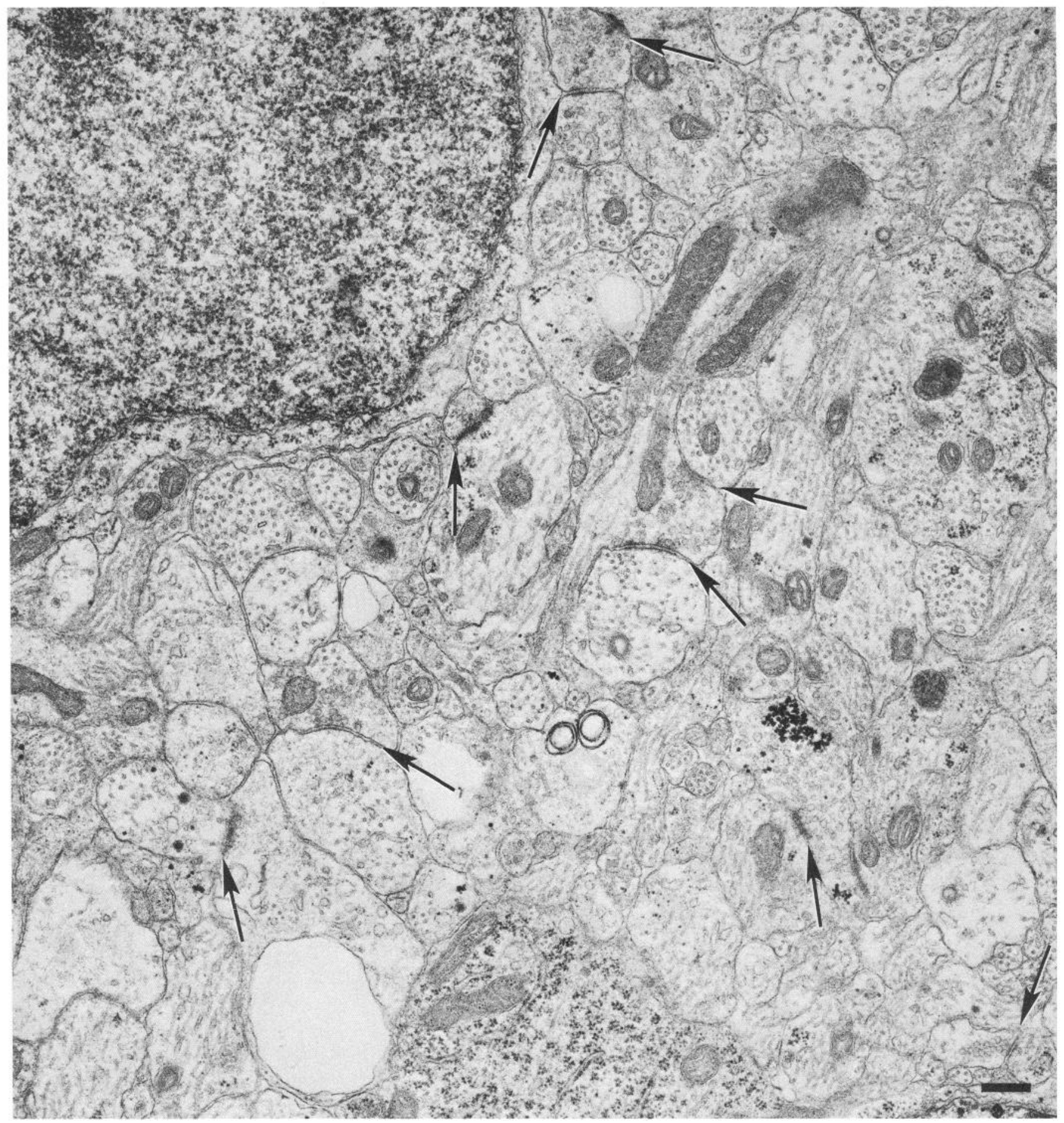

Figure 3. Electron micrograph showing SCN ultrastructure at P4. There are a number of axon terminals making synaptic contacts with dendritic profiles (arrows), but the neuropil is immature compared with later developmental stages. Scale bar, $2 \mu \mathrm{m}$.

These begin to appear between P6 and P10 but are not present in large numbers.

\section{Synaptogenesis}

At E19 there are only very rare synaptic profiles present, and these are immature with very few synaptic vesicles. From E21 to $\mathrm{P} 6$ there is a gradual increase in synaptic number and synaptic diversity (Fig. 4). The initial synapses formed are on medium to large dendritic profiles by small axon terminals containing a few spherical vesicles clustered near the synaptic contact. Between E19 and P2, synaptogenesis proceeds slowly and the number of axon terminals present at P2 is only about $10 \%$ of that in the adult. There is a rapid increase in the number of terminals between $\mathrm{P} 2$ and P6, but the most active period of synaptogenesis is between P6 and P10, when the number of axon terminals per unit area of SCN is the same as in the adult (Fig. 8). In addition, 

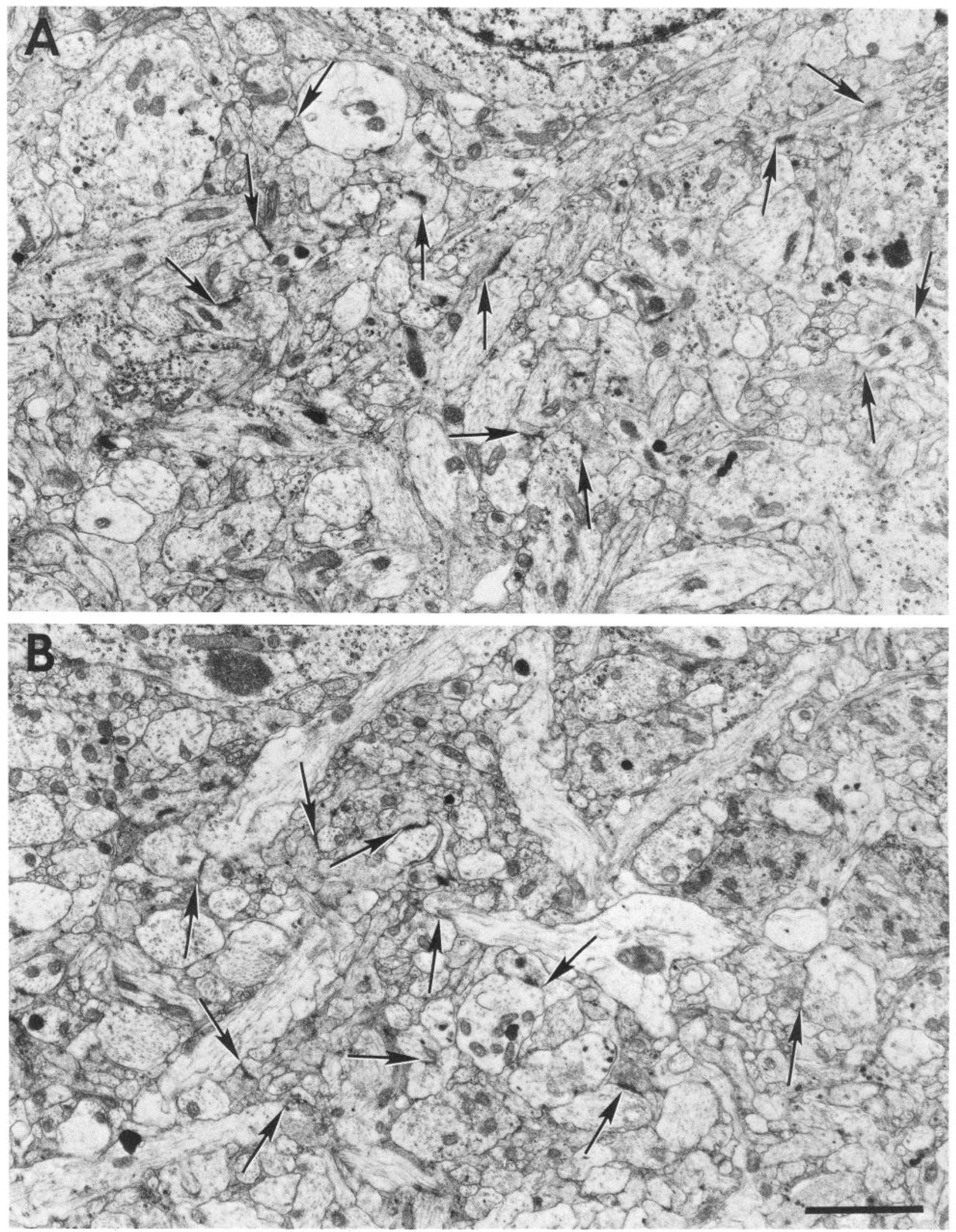

Figure 4. Electron micrographs showing the ultrastructure of 2 areas of SCN neuropil at P10. The neuropil is very complex, with many small axons and dendrites and numerous synaptic contacts (arrows). Scale bar, $2 \mu \mathrm{m}$. 


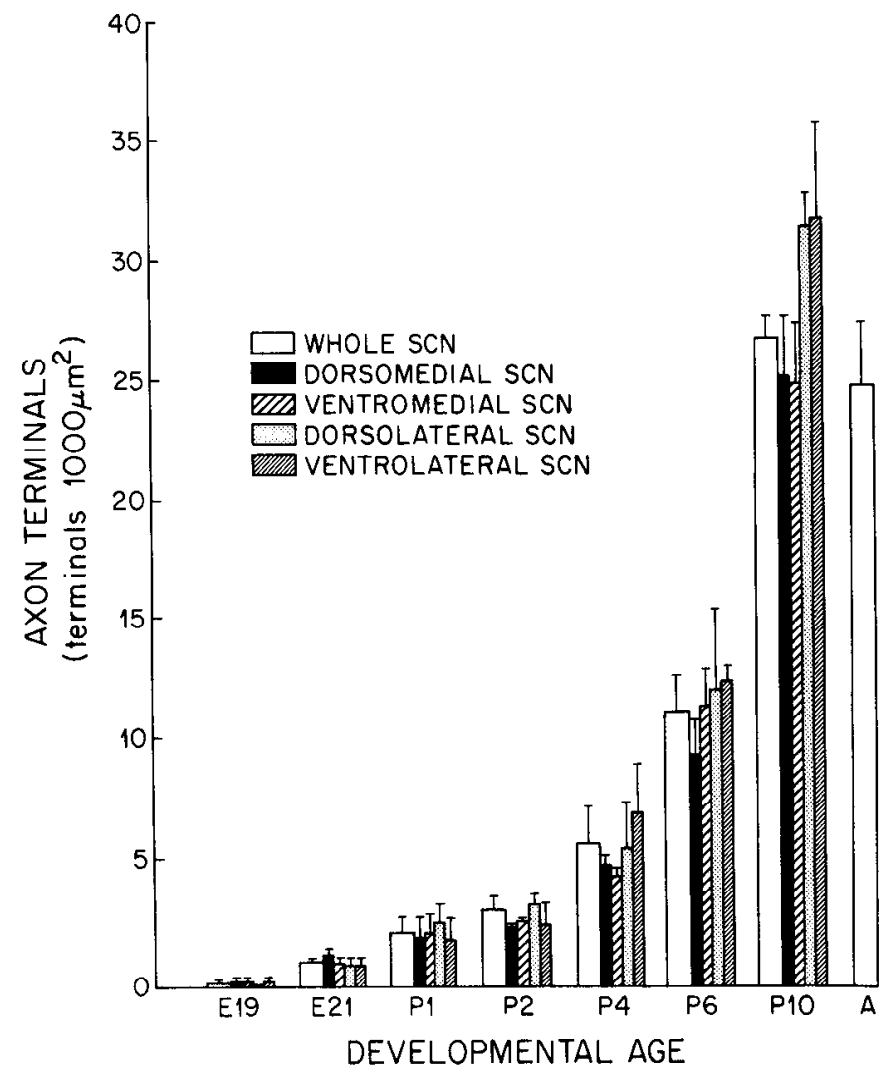

Figure 5. Synaptogenesis in the SCN shown by bar graphs of synaptic counts recorded for whole SCN and for each of 4 SCN quadrants: dorsomedial, ventromedial, dorsolateral, and ventrolateral.

at P10 all of the types of axon terminal morphology are present; also, all forms of synaptic contact are evident except axoaxonal synapses, but these are very rare in the adult. The time course of development of specific axon terminal types and types of synaptic contact are shown in Table 1. Only the lucent terminals with small spherical vesicles and "tubular" mitochondria have been characterized with absolute certainty at this time. As noted by Güldner $(1978 \mathrm{a}, \mathrm{b})$, these are the terminals of the retinohypothalamic tract. It is of interest that they go through a phase of maturation after first appearing at $\mathrm{P} 4$, about the time retinohypothalamic projections are noted with light microscopy (Stanfield and Cowan, 1976; Mason et al., 1977). The first terminals to innervate the SCN, at P4 and P6, are small with fewer vesicles than in the adult, but by P10 they are nearly all large with numerous round vesicles. The origin of other terminal types is not known but many undoubtedly represent intrinsic innervation. It also seems likely that a particular terminal type may arise from more than one source.

The quantitative analysis of synaptogenesis shown in Figure 5 not only illustrates the very rapid postnatal synaptogenesis between P6 and P10 for the whole SCN but shows some interesting regional variation within the nucleus. Early axon terminal formation is fairly even across the nucleus, but at P4 there is an abrupt increase in the ventrolateral quadrant that marks the development of the retinal afferents into that area. From P4 on, the number of synapses in the 2 medial quadrants is lower than the number in the 2 lateral quadrants. This reflects the fact that the neurons in the medial portion of the nucleus are more closely packed, and the total neuropil in this part of the nucleus, the "nonvisual" component, is less than in the "visual" component.

The development of the interface between the SCN and the optic chiasm has several special features. At E19 and E21 there is a sharp boundary between the chiasm and the ventral border of the SCN. Numerous growth cones are evident in the chiasm but no axons enter the SCN. Between P1 and P4 there is a gradual intercalation of elements of the chiasm with components of the ventral portion of the SCN. Fascicles of axons are mixed among neuronal perikarya, and developing pockets of neuropil appear between P4 and P6. Dendrites extend into the chiasmal bordcr, and by P6 there is extensive synapse formation. At this time there is no clear border between the chiasm and the SCN in electron micrographs. However, at P10 the initiation of myelination of optic chiasm axons permits a good approximation of the ventral limits of the SCN. It should be noted that the pockets of neuropil in the dorsal chiasm-SCN interface contain retinohypothalamic terminals and several of other terminal types. Dendrodendritic synapses (Güldner and Wolff, 1974) are most readily found in the zone adjacent to and within the chiasm.

The general pattern of development of synaptic development shown by the quantitative ultrastructural analysis is confirmed by the analysis of the development of synapsin I immunoreactivity (SI-IR). The immunohistochemical analysis has the further advantage of allowing a comparison of synaptic development in the SCN with other hypothalamic nuclei and, particularly, those immediately adjacent to the SCN. SI-IR appears as small punctate structures that we interpret to represent axon terminals (Figs. 6, 7). SI-IR is present at E19 in the lateral hypothalamic area, the paraventricular nucleus, and the anterior hypothalamic area adjacent to the SCN, but there are no immunoreactive structures within the SCN itself. There appears to be a general gradient of SI-IR with the lateral hypothalamus most heavily innervated and medial structures less so. The SIIR within the paraventricular nucleus is quite dense, howevcr. A few SI-IR structures are present in the SCN at E21, but this is much less than in the surrounding hypothalamus. From E21 and $\mathrm{P} 2$, there is a rapid increase in the number of SI-IR structures in the anterior and lateral hypothalamic areas and only a modest increase in the number in the SCN (Fig. 7). At P4 there is a large increase in SI-IR in the ventrolateral quadrant of the $\mathrm{SCN}$, and between P4 and P10 the innervation of the nucleus approximates the adult pattern. SI-IR appears identical in P10 and adult material. The dorsomedial division of the nucleus shows less SI-IR than the ventrolateral division, as is evident in the quantitative ultrastructural data. Thus, there is almost complete agreement between the quantitative ultrastructural analysis and synapsin immunohistochemistry with respect to the timing of synaptogenesis in the SCN. The only significant disparity is that the density of innervation appears more nearly like the adult at $\mathrm{P} 6$ in the synapsin material than indicated by the quantitative analysis. The development of SI-IR in the SCN lags considerably behind that of adjacent hypothalamic nuclei. This is not simply an expression of a lateral to medial gradient of synapse formation, however, as the periventricular nucleus shows dense SI-IR development prior to the SCN and it is more medially located. The general pattern of synapse formation as shown by SI-IR is illustrated diagrammatically in Figure 8 .

\section{Discussion}

Neurons of the SCN are formed in the third wave of hypothalamic neurons as described in tritiated thymidine material by 

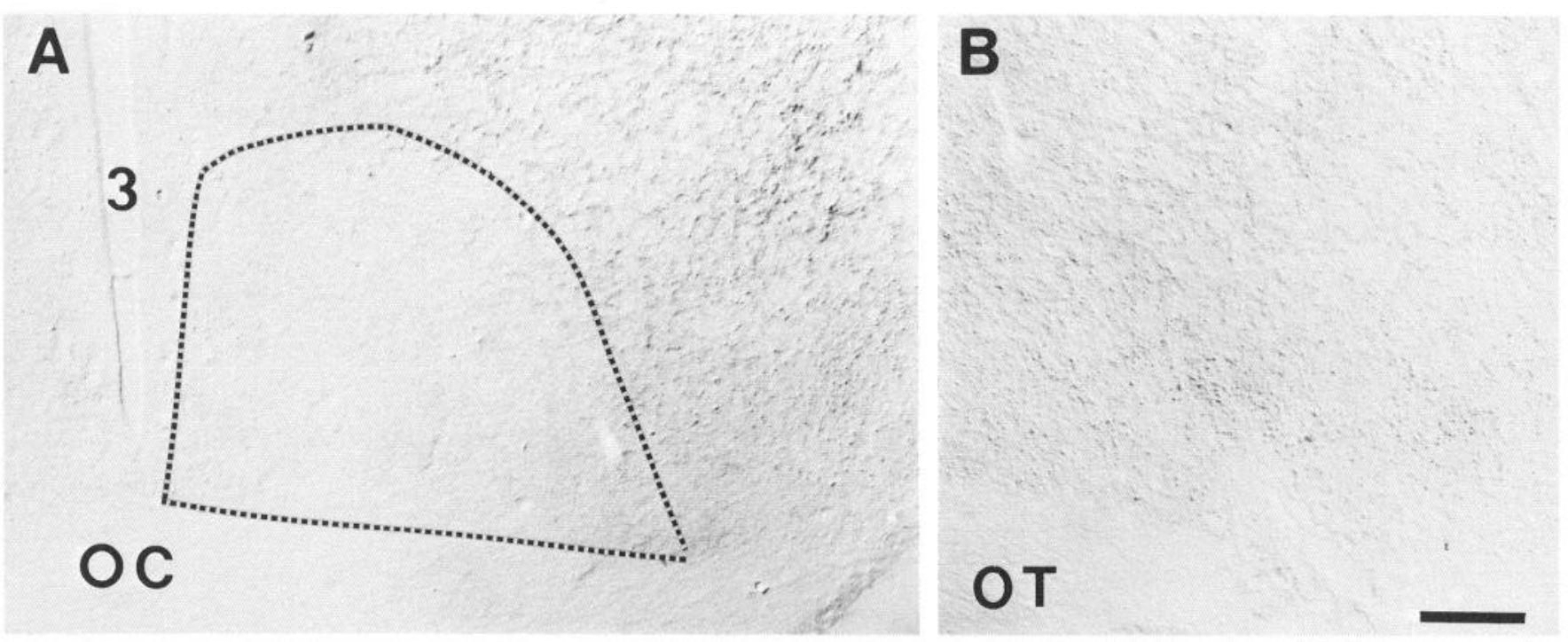

Figure 6. Photomicrographs of synapsin I immunoreactivity at E19. A, SCN showing very few immunoreactive structures. The border of the nucleus is outlined by an interrupted line. $O C$, optic chiasm; 3 , third ventricle. $B$, Lateral hypothalamus showing numerous immunoreactive structures above and lateral to the optic tract $(O T)$. Scale bar, $100 \mu \mathrm{m}$.

Altman and Bayer (1986). The lateral hypothalamic area and related structures are formed in the first wave followed by the major medial hypothalamic nuclei in the second. The SCN is formed between E13 and E17, with the majority of neurons generated on E15-E17, at the same time as the formation of the periventricular field and the arcuate nucleus. The first neurons to be formed in the SCN are those of the ventrolateral division, followed by the dorsomedial division, and then neurons lying along the ventral surface of the nucleus (Altman and Bayer, 1986). The morphometric data from the present study indicate that the $\mathrm{SCN}$ increases in length and volume at an almost steady rate from E19 to P10 and continues to increase in size to the adult. Our measurement of adult volume $\left(0.037 \mathrm{~mm}^{3}\right)$ is within the general range of recent reports. Güldner (1983) reported a volume of $0.064 \mathrm{~mm}^{3}$, van den Pol (1980), $0.068 \mathrm{~mm}^{3}$; and
Robinson et al. (1986), $0.029 \mathrm{~mm}^{3}$ for the male and $0.023 \mathrm{~mm}^{3}$ for the female. The differences among these studies probably reflect differences in tissue preparation and slightly different criteria for defining the boundary of the nucleus.

The general ultrastructural features of the development of the nucleus are similar to those that have been reported previously (Lenn et al., 1977; Koritsanszky, 1981). Our study differs from those in providing a quantitative ultrastructural analysis of synaptogenesis and a correlation of this with the pattern of synaptogenesis demonstrated by synapsin I immunohistochemistry. This has been done previously in the cerebellum by Mason (1986). Synapsin I is a specific neuronal protein that is a substrate for protein kinases (Johnson et al., 1972; Nestler and Greengard, 1984). It is found throughout the nervous system, particularly at synapses (Bloom et al., 1979; DeCamilli et al.,

Table 1. Time course of development of axon terminal types and synaptic configurations in the SCN

\begin{tabular}{llllllll} 
& \multicolumn{3}{l}{ Developmental age } & & & \\
\cline { 2 - 7 } Terminal type/synaptic configuration & E19 & E21 & P1 & P2 & P4 & P6 & P10 \\
\hline Lucent/small spherical & $*$ & $*$ & $*$ & $* *$ & $* * *$ & $* * *$ & $* * * *$ \\
Lucent/small pleomorphic with LDCVs & & $*$ & $*$ & $* *$ & $* *$ & $* * *$ & $* * * *$ \\
Lucent/small pleomorphic & & & $*$ & $* *$ & $* *$ & $* * *$ & $* * * *$ \\
Lucent/large pleomorphic & & & & & & $*$ & $* * *$ \\
Lucent/small spherical with "tubular" mitochondria & & & & $*$ & $* *$ & $* * * *$ \\
Dense/small spherical & & & $*$ & $* *$ & $* *$ & $* * *$ & $* * * *$ \\
Dense/small pleomorphic with LDCVs & & & & & & & $* *$ \\
Axodendritic contacts & $*$ & $*$ & $*$ & $* *$ & $* *$ & $* * *$ & $* * * *$ \\
Axospinous contacts & & $*$ & $*$ & $*$ & $* *$ & $* * *$ & $* * * *$ \\
Dendrodentritic contacts & & & & & $*$ & $* *$ & $* * *$ \\
Axosomatic contacts & & & & & & & $* *$
\end{tabular}

Terminal type is characterized by the density of terminal cytoplasm (lucent or dense) and the morphology of synaptic vesicles. Synaptic configuration is specified. The asterisk designations are rough approximations of the number of structures present at any developmental age, with a single symbol representing scarce and four symbols being equivalent to the adult pattern. LCDVs are large dense-core vesicles. A terminal was characterized as containing LDCVs if more than 2 were present in a profile. 

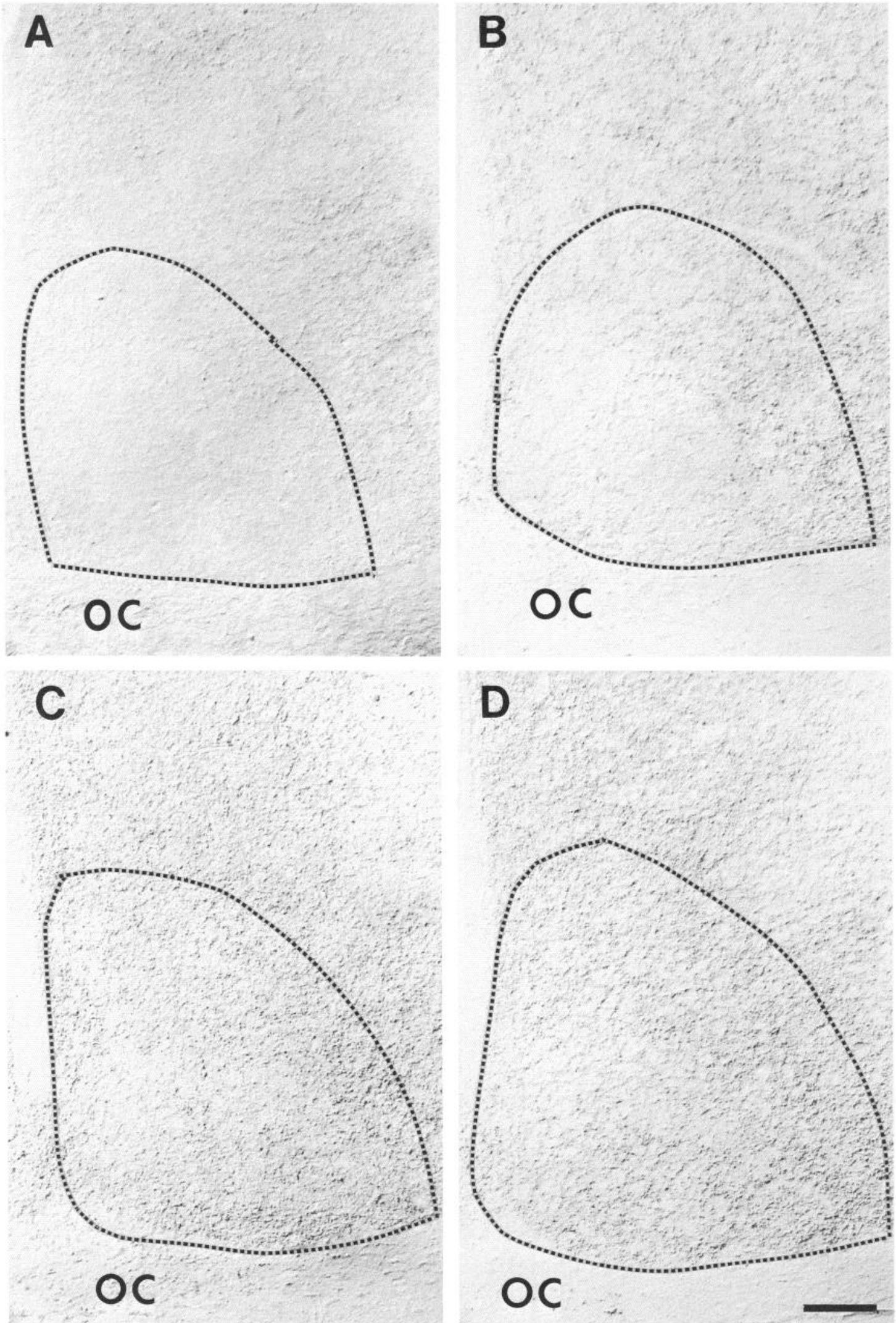

Figure 7. Photomicrographs of synapsin I immunoreactivity: $A, \mathrm{E} 21 ; B, \mathrm{Pl} ; C, \mathrm{P} 4 ; D, \mathrm{P} 6$. Abbreviations as for Figure 6 . Scale bar, $100 \mu \mathrm{m}$. 


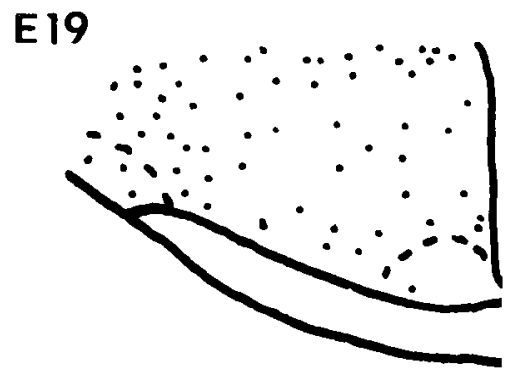

E21
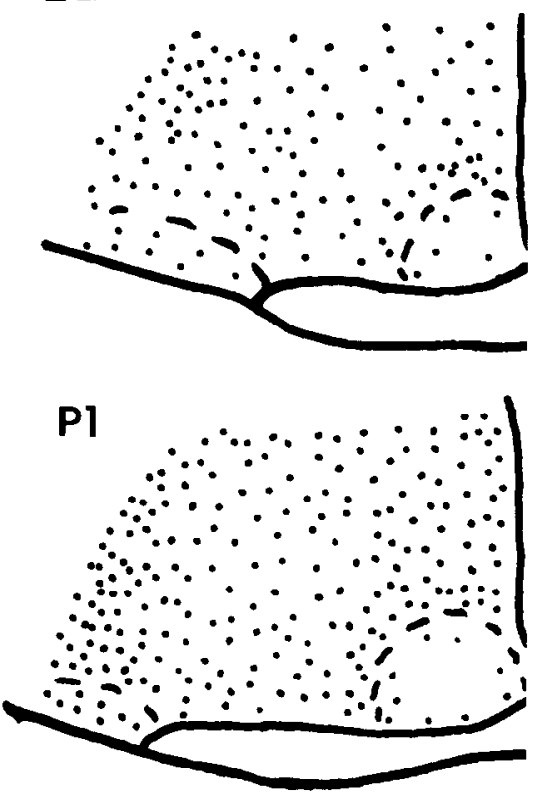

P4
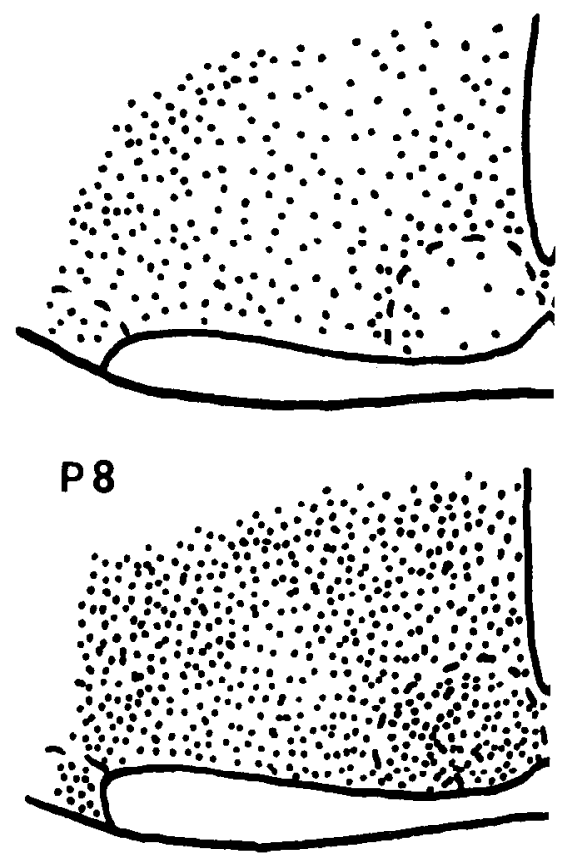

Figure 8. Drawings showing the pattern of synapsin I immunoreactivity in the SCN and adjacent hyothalamus at E19, E21, P1, P4, and P8.

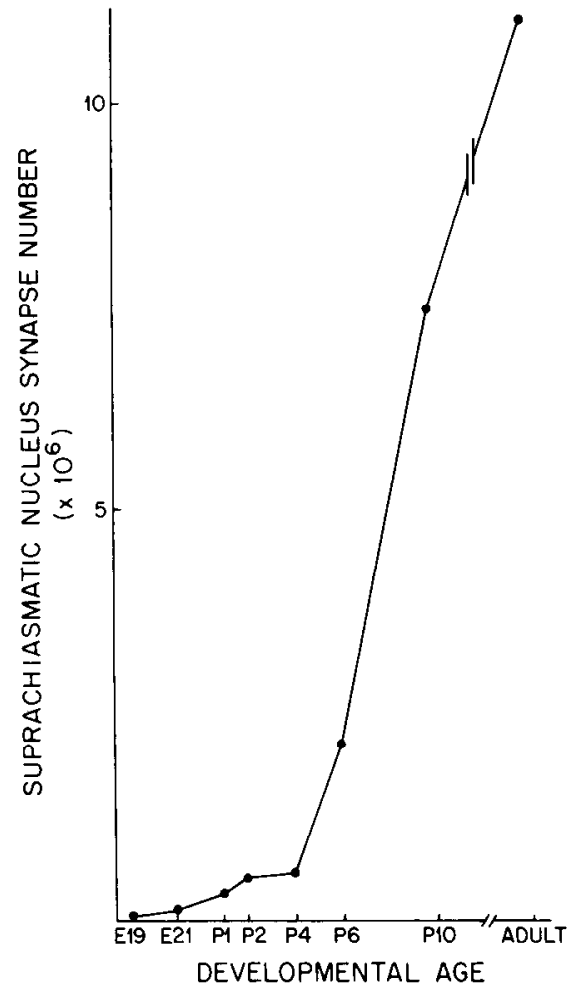

Figure 9. Synaptogenesis in the $\mathrm{SCN}$ is illustrated by plotting total SCN synapse number at each developmental age. Total synapse number was estimated by taking the mean values for number of axon terminals per $1000 \mu \mathrm{m}^{2}$ and calculating a number per $1000 \mu \mathrm{m}^{3}$ using an estimated ultra thin section thickness of $80 \mathrm{~nm}$. This was multiplied by the volume of the nucleus at each age.

1979, 1983a) and appears to be a component of the vesicle membrane of small synaptic vesicles (Huttner et al., 1983; Navone et al., 1984). Both synapsin I and its mRNA appear during development at the time synapses are being formed (Lohman et al., 1979; Haas and DeGennaro, 1988). Thus, it seems likely that SI-IR would be a reliable marker for synapse development, but this has not been tested prior to the present study. Our data indicate that the development of SI-IR is a very accurate marker for synapse formation, at least early in the course of synapse development. When an area has a large complement of synapses, it is difficult to appreciate any further changes. It is possible that this could be done by radioimmunoassay, but in doing so the morphological information provided by immunohistochemistry would be lost. Nevertheless, there is a remarkably close correlation in this study between the number of synapses present in the ultrastructural material and the synapsin immunoreactive structures indicating that synapsin immunohistochemistry could be a valuable method for studying synaptogenesis. It is noteworthy in our material, for example, that the development of SI-IR in the hypothalamic areas adjacent to the SCN occurs much more rapidly than in the SCN. There are differences among these areas indicating the utility of SI-IR for demonstrating patterns of synaptogenesis in brain.

Although it is possible to characterize the development of specific synaptic terminal types and types of synaptic contacts, this provides relatively little information at the present time because only the terminals of the retinohypothalamic projection (Güldner, 1978a, b; Güldner and Wolff, 1978) have been definitively identified among the number of synaptic terminal types 
E19

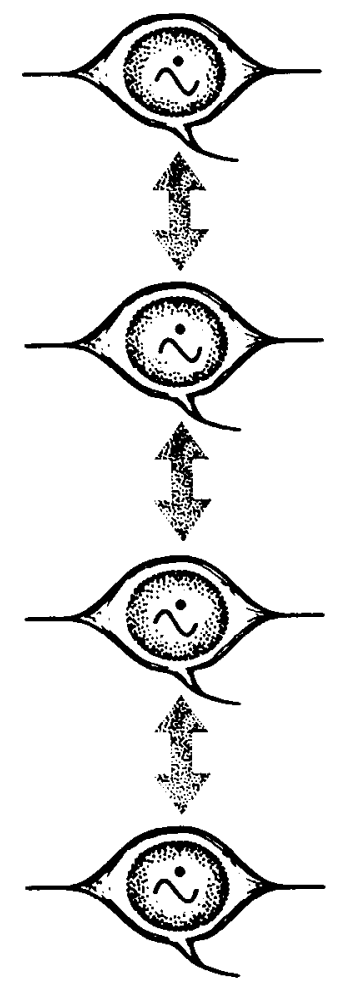

P10

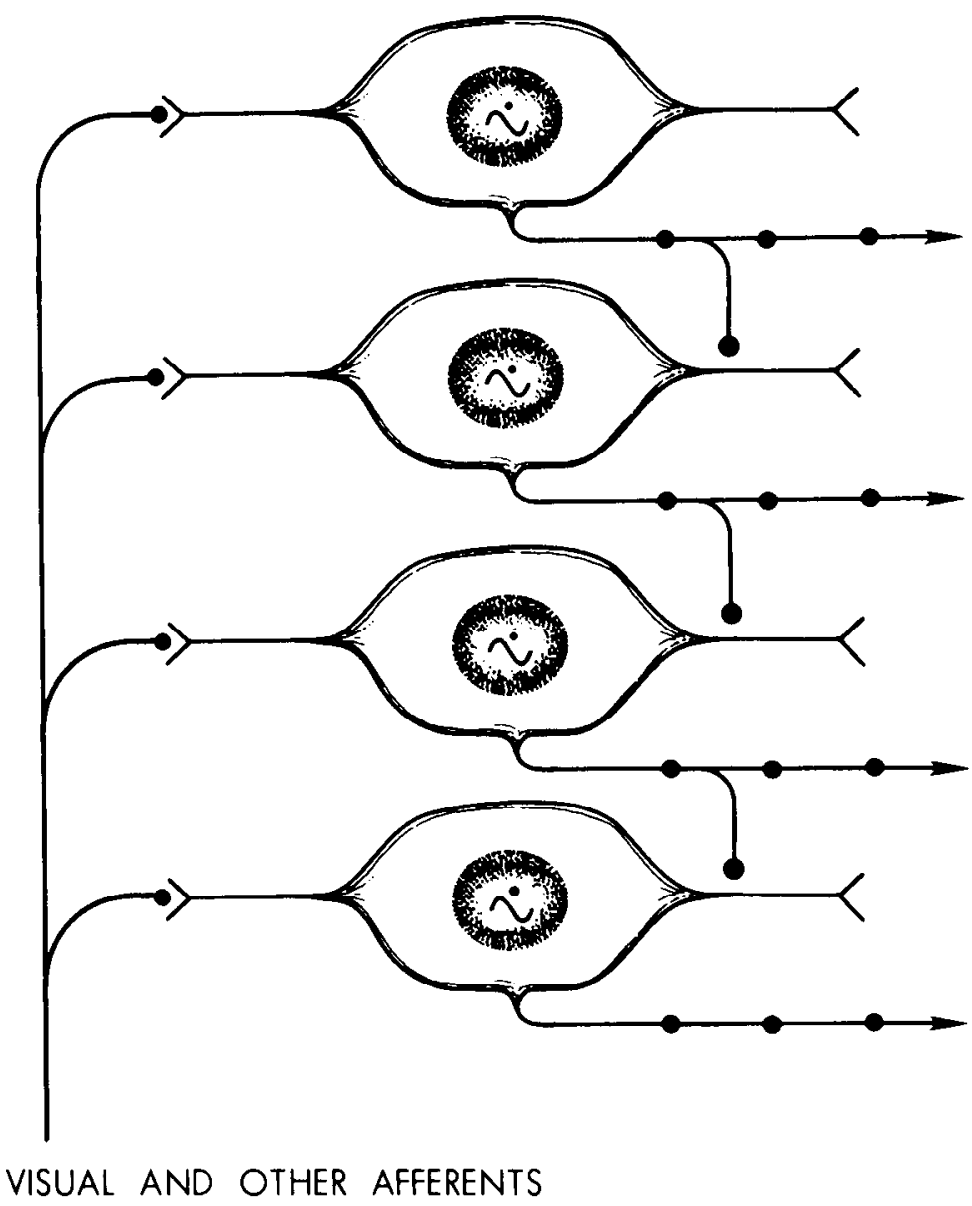

Figure 10. Diagrammatic representation of the development of the SCN from E19 to P10. At E19 the neurons are small with few proceses and essentially no connections. Interaction among neurons must occur by a process that is not known, represented by stippled arrows. At P10 the SCN is a well-developed neural network with mature neurons having afferent, efferent, and intrinsic connections. The sine wave $(\sim)$ in the nucleus of each neuron conveys the hypothesis that SCN ncurons are born as circadian oscillators.

present in the SCN (Güldner, 1976, 1984; van den Pol, 1980). Data from both Golgi analysis (van den Pol, 1980) and immunohistochemistry (Card et al., 1981; van den Pol and Tsujimoto, 1985) indicate that SCN neurons produce large numbers of connections that are intrinsic to the nucleus. This suggests that many of the synaptic types evident in ultrastructural material are from SCN neurons.

The process of SCN development appears to have 3 phases: neuron formation, neuron growth, and maturation and synapse formation. All SCN neurons are formed prenatally and, as noted above, predominantly before E18 (Seress, 1985; Altman and Bayer, 1986). Our morphometric data and the light and electron microscopic analysis of $\mathrm{SCN}$ development demonstrate that the general maturation of the nucleus progresses steadily from the time the neurons are formed to $\mathrm{P} 10$ and continues to the adult. Indeed, even though the number of synapses per unit $\mathrm{SCN}$ area is the same at P10 and in the adult, the volume of the nucleus is significantly larger in the adult and this can only be explained by a continuing extension of neuropil, particularly with the development of small dendritic processes and their innervation by intrinsic and extrinsic afferents already present in the nucleus. Finally, synapse formation progresses very slowly in the late prenatal and early postnatal periods and then markedly increases from P4 to P10. This is illustrated in Figure 9, which plots estimated total SCN synapse number against developmental age. The estimates are undoubtedly crude, but there should not be any inherent inaccuracy that would affect the estimate at any particular developmental ages. The estimated synapse number for the adult $-11,000,000-$ is within the range that would be predicted from Güldner's work. Güldner (1976) calculated that each SCN neuron would have 300-1200 synaptic contacts. The total number of SCN neurons is approximately 10,000 (Riley and Moore, 1977; van den Pol, 1980; Güldner, 1983), so that our estimate of synapse number is within the range, albeit toward the high end, that would be predicted from Güldner's data. Figure 9 clearly illustrates the striking increase in synapse number occurring between P6 and P10 and demonstrates that approximately $30 \%$ of total synapse number is added after P10. Synaptic density is less in the SCN than has been described for other areas (Cragg, 1975), probably reflecting the close neuronal packing of the nucleus and the relative segregation of synapses to small dendrites.

The functional implications of these observations are particularly interesting. At E19 there are few synapses, and the esti- 
mate of total synapses at that age, approximately 9000 , is about one synapse per neuron. Nevertheless, the SCN at E19 exhibits a rhythm in glucose metabolism (Reppert and Schwartz, 1983, 1984) that is entrained to maternal rhythmicity by a mechanism not yet fully elucidated (Reppert, 1985; Reppert and Schwartz, 1986). The development of the rhythm is not dependent upon maternal rhythmicity (Reppert and Schwartz, 1986; Shibata and Moore, 1988). The earliest rhythms known to be expressed are within the SCN, the rhythm in glucose metabolism (Fuchs and Moore, 1980; Reppert and Schwartz, 1983, 1984) and the rhythm in firing rate of SCN neurons (Shibata and Moore, 1987). The rhythm in firing rate is present at E22 but is of very low amplitude, and its subsequent development correlates well with SCN development. Like the rhythm in glucose metabolism, it develops in a very immature nucleus with very few synapses. These observations indicate that the SCN expresses circadian function at a time prior to the development of significant intrinsic or extrinsic connections. At this stage the SCN is a circadian oscillator, and we would offer the hypothesis that SCN neurons are born as individual oscillators that are initially coupled by non-neural signals among themselves and from the mother (Fig. 10). The SCN becomes a circadian pacemaker when it develops sufficient afferent, intrinsic and efferent connections to permit it to function as a neural network. This is accomplished by P4-P6, when a rhythm in the enzyme, serotonin $N$-acetyltransferase, develops and is entrained to the lightdark cycle (Klein et al., 1981; Duncan et al., 1986). There is a continuing maturation of the nucleus thereafter, but it seems most likely that the long postnatal period during which the development of circadian rhythms occurs (see Davis, 1981, for review) reflects the functional development of systems that express circadian rhythms rather than development of the SCN and its connections.

In summary, the SCN develops circadian rhythms prenatally, shortly after its formation, at a time when the nucleus is quite immature. Its synaptic development is largely a postnatal event with a spurt of synaptogenesis occurring between P4 and P10. Synapsin I immunohistochemistry reveals a time course for synaptogenesis that correlates well with quantitative ultrastructural analysis. This indicates that the appearance of synapsin I is a precise indicator of the time of synapse formation and that synapsin I immunohistochemistry provides a valuable tool for the study of synaptogenesis.

\section{References}

Altman, J., and S. A. Bayer (1978) Development of the diencephalon in the rat. I. Autoradiographic study of the time of origin and settling patterns of neurons of the hypothalamus. J. Comp. Neurol. 182: 945972.

Altman, J., and S. A. Bayer (1986) The development of the rat hypothalamus. Adv. Anat. Embryol. Cell Biol. 100: 1-178.

Bloom, F. E., T. Ueda, E. Battenberg, and P. Greengard (1979) Immunocytochemical localization in synapses of Protein $I$, an endogenous substrate for protein kinases in mammalian brain. Proc. Natl. Acad. Sci. USA 76: 5982-5986.

Card, J. P., N. Brecha, H. J. Karten, and R. Y. Moore (1981) Immunocytochemical localization of vasoactive intestinal polypeptide containing cells and processes in the suprachiasmatic nucleus of the rat: Light and electron microscopic analysis. J. Neurosci. 1: 1289 1303.

Cragg, B. G. (1975) The development of synapses in the visual system of the cat. J. Comp. Neurol. 160: 147-166.

Davis, F. C. (1981) Ontogeny of circadian rhythms. In Handbook of Behavioral Neurobiology, Vol. 4, Biological Rhythms, J. Aschoff, ed., pp. 257-276, Plenum, New York.
DeCamilli, P., T. Ueda, F. E. Bloom, E. Battenberg, and P. Greengard (1979) Widespread distribution of Protein I in the central and peripheral nervous system. Proc. Natl. Acad. Sci. USA 76: 5977-5981.

DeCamilli, P., R. Cameron, and P. Greengard (1983a) Synapsin I (Protein I), a nerve terminal specific phosphoprotein. I. Its general distribution in synapses of the central and peripheral nervous system demonstrated by immunofluorescence in frozen and plastic sections. J. Cell Biol. 96: 1337-1354.

DeCamilli, P., S. M. Harris, W. B. Huttner, and P. Greengard (1983b) Synapsin I (Protein I), a nerve terminal-specific phosphoprotein. II. Its specific association with synaptic vesicles demonstrated by immunocytochemistry in agarose-embedded synaptosomes. J. Cell Biol. 96: 1355-1373.

Duncan, M. J., M. J. Banister, and S. M. Reppert (1986) Developmental appearance of light-dark entrainment in the rat. Brain Res. 369: 326-330.

Fuchs, J. L., and R. Y. Moore (1980) Development of circadian rhythmicity and light responsiveness in the rat suprachiasmatic nucleus: A study using the 2-deoxy(1-14C) glucose method. Proc. Natl. Acad. Sci. USA 77: 1204-1208.

Güldner, F.-H. (1976) Synaptology of the rat suprachiasmatic nucleus. Cell Tissue Res. 165: 509-544.

Güldner, F.-H. (1978a) Synapses of optic nerve afferents in the rat suprachiasmatic nucleus. I. Identification, qualitative description, development and distribution. Cell Tissue Res. 194: 17-35.

Güldner, F.-H. (1978b) Synapses of optic nerve afferents in the rat suprachiasmatic nucleus. II. Structural variability as revealed by morphometric examination. Cell Tissue Res. 194: 37-54.

Güldner, F.-H. (1983) Numbers of neurons and astroglial cells in the suprachiasmatic nucleus of male and female rats. Exp. Brain Res. 50: 373-376.

Güldner, F.-H. (1984) Suprachiasmatic nucleus: Numbers of synaptic appositions and various types of synapses. Cell Tissue Res. 235:449452.

Güldner, F.-H., and J. R. Wolff (1974) Dendro-dendritic synapses in the suprachiasmatic nucleus of the hypothalamus. J. Neurocytol. 3 : 245-250.

Güldner, F.-H., and J. R. Wolff (1978) Retinal afferents form GrayType-I and Type-II synapses in the suprachiasmatic nucleus Exp. Brain Res. 32: 83-89.

Haas, C. A., and L. J. DeGennaro (1988) Multiple synapsin I messenger RNA's are differentially regulated during neuronal development. J. Cell Biol. 106: 195-203.

Huttner, W. B., W. Schrebler, P. Greengard, and P. DeCamilli (1983) Synapsin I (Protein I), a nerve terminal-specific phosphoprotein. III. Its association with synaptic vesicles studied in a highly purified synaptic vesicle preparation. J. Cell. Biol. 96: 1374-1388.

Ifft, J. D. (1972) An autoradiographic study of the time of final division of neurons in the rat hypothalamic nuclei. J. Comp. Neurol. 144 . 193-204.

Johnson, E. M., T. Ueda, H. Maeno, and P. Greengard (1972) Adenosine 3', 5'-monophosphate dependent phosphorylation of a specific protein in synaptic membrane fractions from rat cerebrum. J. Biol. Chem. 247: 5650-5652.

Klein, D. C., M. A. A. Namboodiri, and D. A. Aurbach (1981) The melatonin rhythm generating system. Life Sci. 28: 1975-1986.

Koritsanszky, S. (1981) Fetal and early postnatal cyto- and synaptogenesis in the suprachiasmatic nucleus of the rat hypothalamus. Acta Morphol. Acad. Sci. Hung. 29: 227-239.

Lenn, N. J., B. Beebe, and R. Y. Moore (1977) Postnatal development of the suprachiasmatic hypothalamic nucleus in the rat. Cell Tissue Res. 178: 463-475.

Lohman, S. M., 'T. Ueda, and P. Greengard (1979) Ontogeny of synaptic phosphoproteins in brain. Proc. Natl. Acad. Sci. USA 75:40374041.

Mason, C. A. (1986) Axon development in mouse cerebellum. Embryonic axon forms and expression of synapsin I. Neuroscience 19: $1319-1333$.

Mason, C. A., N. Sparrow, and D. W. Lincoln (1977) Structural features of the retinohypothalamic projection in the rat during normal development. Brain Res. 132: 141-148.

Moore, R. Y. (1983) Organization and function of a central nervous system circadian oscillator: The suprachiasmatic hypothalamic nucleus. Fed. Proc. 42: 2783-2789.

Navone, F., P. Greengard, and P. DeCamilli (1984) Synapsin I in 
nerve terminals: Selective association with small synaptic vesicles. Science 226: 1209-1211.

Nestler, E. J., and P. Greengard (1984) Protein Phosphorylation in the Nervous System, Wiley Interscience, New York.

Reppert, S. M. (1985) Maternal entrainment of the developing circadian system. Ann. NY Acad. Sci. 453: 162-169.

Reppert, S. M., and W. J. Schwartz (1983) Maternal coordination of the fetal biological clock in utero. Science 220:969-971.

Reppert, S. M., and W. J. Schwartz. (1984) The suprachiasmatic nuclei of the fetal rat: Characterization of a functional circadian clock using 14C-labeled deoxyglucose. J. Neurosci. 4: 1677-1682.

Reppert, S. M., and W. J. Schwartz (1986) Maternal suprachiasmatic nuclei are necessary for coordination of the developing ciradian system. J. Neurosci. 6: 2724-2729.

Riley, J. N., and R. Y. Moore (1977) Organization of the suprachiasmatic nucleus in the albino rat. Soc. Neurosci. Abstr. 3: 355

Robinson, S. M., T. O. Fox, P. Dikkes, and R. A. Pearlstein (1986) Sex difterences in the shape of the sexually dimorphic nucleus of the preoptic area and the suprachiasmatic nucleus of the rat: 3-D computer reconstructions and morphometrics. Brain Res. 371: 380-384.

Rosenwasser, A. M., and N. T. Adler (1986) Structure and function in circadian timing systems: Evidence for multiple coupled circadian oscillators. Neurnsci. Behav. Rev. 10: 431-448.

Rusak, B., and I. Zucker (1979) Neural regulation of circadian rhythms. Physiol. Rev. 59: 449-526.

Schwartz, W. J., and H. Gainer (1977) Suprachiasmatic nucleus: Use of $14 \mathrm{C}$-labeled deoxyglucose uptake as a functional marker. Science 197: 1089-1091.
Schwartz, W., L. C. Davidson, and C. B. Smith (1980) In vivo metabolic activity of a putative circadian oscillator, the rat suprachiasmatic nucleus. J. Comp. Neurol. 189: 157-167.

Seiger, A., and L. Olson (1973) Late prenatal ontogeny of central monoamine neurons in the rat: Fluorescence histochemistry. Z. Anat. Entwickl. Gesch. 140: 281-318.

Seress, L. (1985) Postnatal neurogenesis in the rat hypothalamus. Dev. Brain Res. 22: 156-160.

Shibata, S., and R. Y. Moore (1987) Development of neuronal activity in the rat suprachiasmatic nucleus. Dev. Brain Res. 34: 311-315.

Shibata, S., and R. Y. Moore (1988) Development of a fetal circadian rhythm after disruption of the maternal circadian system. Dev. Brain Res. 41: 313-317.

Sokoloff, L. (1982) The radioactive deoxyglucose method. Theory procedure and applications for the measurement of local glucose utilization in the central nervous system. Adv. Neurochem. 4: 1-82.

Stanfield, B., and W. M. Cowan (1976) Evidence for a change in the retino-hypothalamic projection in the rat following early removal of on eye. Brain Res. 104: 129-136.

Turek, F. W. (1985) Circadian neural rhythms in mammals. Annu. Rev. Physiol. 47: 49-64.

van den Pol, A. N. (1980) The hypothalamic suprachiasmatic nucleus: Intrinsic anatomy. J. Comp. Neurol. 191: 661-702.

van den Pol, A. N., and K. Tsujimoto (1985) Neurotransmitters of the hypothalamic suprachiasmatic nucleus-immunocytochemical analysis of 22 neuronal antigens. Neuroscience 15: 1049-1086. 